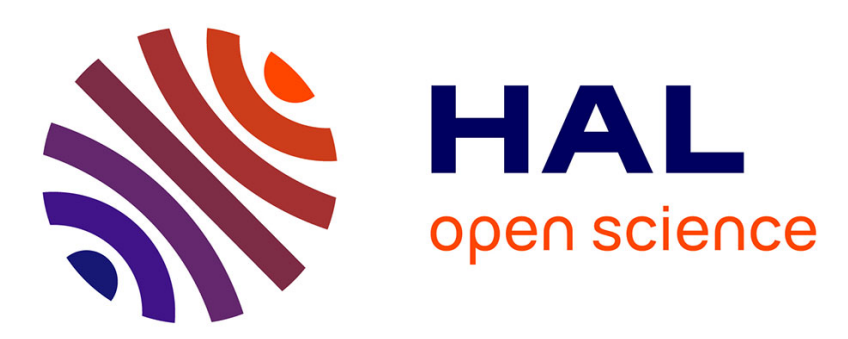

\title{
Modélisation de la réponse d'une antenne d'hydrophones surfaciques aux vibrations d'une structure raidie
}

\author{
A. Flori, J. Houdebine
}

\section{To cite this version:}

A. Flori, J. Houdebine. Modélisation de la réponse d'une antenne d'hydrophones surfaciques aux vibrations d'une structure raidie. Journal de Physique IV Proceedings, 1994, 04 (C5), pp.C5-335-C5338. 10.1051/jp4:1994569 . jpa-00253064

\section{HAL Id: jpa-00253064 https://hal.science/jpa-00253064}

Submitted on 1 Jan 1994

HAL is a multi-disciplinary open access archive for the deposit and dissemination of scientific research documents, whether they are published or not. The documents may come from teaching and research institutions in France or abroad, or from public or private research centers.
L'archive ouverte pluridisciplinaire HAL, est destinée au dépôt et à la diffusion de documents scientifiques de niveau recherche, publiés ou non, émanant des établissements d'enseignement et de recherche français ou étrangers, des laboratoires publics ou privés. 


\title{
Modélisation de la réponse d'une antenne d'hydrophones surfaciques aux vibrations d'une structure raidie
}

\author{
A. FLORI et J.C. HOUDEBINE
}

ARISTÈ, 25-27 avenue de la Division Leclerc, 92160 Antony, France

Résumé : Les hydrophones surfaciques plongés dans une couche d'élastomère posée sur une coque raidie sont sensibles aux vibrations de cette dernière. Notre problème est d'évaluer leur sensibilité en termes de déformations et par suite de potentiels engendrés aux bornes de leurs armatures. Le modèle proposé nous a permis de tester l'influence de certains des paramètres du système sur les potentiels engendrés aux bornes des hydrophones, à savoir : la position des hydrophones dans la couche d'élastomère, les caracteristiques mécaniques de celui-ci ou encore l'épaisseur des armatures des hydrophones.

\begin{abstract}
The hydrophone plane array in contact with a stiffened shell is sensitive to the vibrations of the latter. A model is presented for the purpose of evaluating strains in the hydrophones and therefore the potential between the armatures when the shell is excited. It allowed us to estimate the influence of some of the parameters of the system as the stiffness of the transducers, their position in the compliant layer and the mechanical features of the layer.
\end{abstract}

\section{INTRODUCTION}

Figure 1. Schéma représentatif du système étudié.

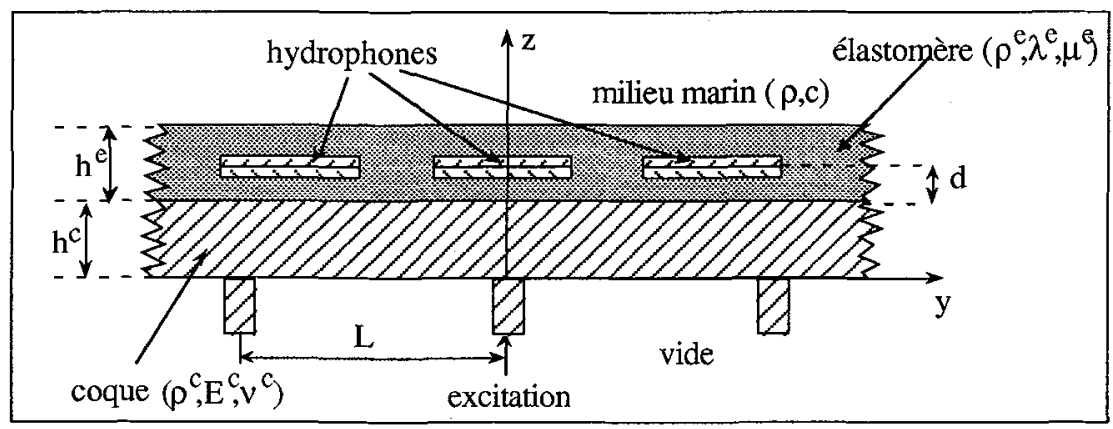

Les hypothèses retenues pour la modélisation du système schématisé sur la figure 1 nous permettent de décomposer l'étude en trois phases successives :

- $1^{\text {ère }}$ phase : Modèle du champ de déplacement dans la coque raidie, recouverte de la couche d'élastomère sans hydrophone, sous l'action d'une force d'excitation ponctuelle directement exercée sur la coque. 
- 2ème phase : Modèle du champ de déplacement et des contraintes subies par les hydrophones, assimilés à des plaques minces présentant les propriétés des hydrophones multicouches homogénéisés, compte tenu du champ de déplacement de la surface supérieure de la coque obtenu précédemment.

- $3^{\text {ème }}$ phase : Modèle du potentiel engendré aux bornes des hydrophones sous l'action des déformations existant au sein des hydrophones.

Les hypothèses et la symétrie du problème : l'extension infinie dans les deux directions $\mathrm{x}$ et $\mathrm{y}$, la répartition périodique des raidisseurs, le caractère purement linéique des contacts entre la coque et les raidisseurs et l'émission monochromatique de la source, nous ont permis d'opter pour une résolution mathématique dans le domaine des nombres d'onde $\mathrm{k}_{\mathrm{x}}$ et $\mathrm{k}_{\mathrm{y}}$.

Le modèle du champ de déplacement dans la coque raidie de la phase 1 ayant fait l'objet de plusieurs publications [1,2], nous renvoyons le lecteur interessé aux travaux mentionnés en référence. Les phases 2 et 3 présentent quant à elles plus d'originalité et font l'objet du paragraphe 2. Dans le dernier paragraphe, nous présentons une application.

\section{DETERMINATION DU CHAMP DE DEPLACEMENT DES HYDROPHONES ASSIMILES A DES PLAQUES MINCES}

Afin de rechercher les caractéristiques mécaniques d'une plaque équivalente à un hydrophone, ce dernier est modélisé par un multicouche plongé dans le vide. L'hydrophone est constitué de deux couches de matériau piézoélectrique enserrées dans des couches d'aluminium représentatives des armatures. Les couches piézoélectriques sont assimilées à des solides élastiques isotropes. Cette hypothèse simplificatrice permet d'aboutir à des caractéristiques équivalentes de plaque mince également isotropes dans le plan de la plaque. Les modes propres de vibration du multicouche correspondant à l'onde de plaque classique (onde à vibrations transversales à la plaque) et à l'onde transversale pour des vibrations dans le plan de la plaque perpendiculaires à la direction de propagation sont associés à ceux d'une plaque mince, ce qui permet d'en déduire les modules d'Young $E^{\text {h }}$ et de Poisson $v^{\text {h }} d^{\prime}$ une plaque équivalente à un hydrophone.

Nous considérons une couche d'élastomère d'extension infinie dans les directions $\mathrm{x}$ et $\mathrm{y}$ dans laquelle sont disposées des plaques minces représentatives des hydrophones et dont les caractéristiques ont été acquises au cours de l'étape précédente. Les plaques carrées de côté 1 sont régulièrement espacées d'une distance $\mathrm{D}$ dans les deux directions $x$ et $y$. On repère les coordonnées du centre d'une plaque de référence par l'abscisse $x_{h}$ et l'ordonnée $y_{h}$. La couche contenant les hydrophones dans l'élastomère est modélisée par une plaque d'épaisseur $\mathrm{h}^{\mathrm{h}}$ dont les caractéristiques mécaniques, $\mathrm{E}^{\mathrm{h}}, v^{\mathrm{h}}$ et la masse volumique $\rho^{\mathrm{h}}$ sont nulles en dehors des hydrophones. Par ailleurs, on fait les hypothèses classiques liées aux plaques minces [3] :

- Les moments des efforts extérieurs sont négligeables.

- Les forces extérieures sont exprimables à partir des contraintes dans l'élastomère exercées de part et d'autre de la couche contenant les hydrophones.

- Les efforts internes sont nuls en dehors de la surface des hydrophones.

- La surface des centres de gravité de la plaque est assimilée à la surface de contact avec l'élastomère.

Dans le domaine des nombres d'onde, les contraintes $\left(\widetilde{\sigma}_{13}^{\mathrm{e}}, \widetilde{\sigma}_{23}^{\mathrm{e}}, \widetilde{\sigma}_{33}^{\mathrm{e}}\right)$ et le déplacement $\widetilde{\vec{u}}_{\mathrm{u}}^{\mathrm{e}}\left(\widetilde{\mathrm{u}}^{\mathrm{e}}, \widetilde{\mathrm{v}}^{\mathrm{e}}, \widetilde{\mathrm{w}}^{\mathrm{e}}\right)$ dans l'élastomère vérifient alors :

$$
\begin{aligned}
& \widetilde{\sigma}_{13}^{e}\left(d_{-}\right)-\widetilde{\sigma}_{13}^{e}\left(d_{+}\right)-\left\{i k_{x} \widetilde{N}_{x}+i k_{y} \widetilde{T}_{y}-\omega^{2} \rho^{h} h^{h} \widetilde{u}^{e}(d)\right\} * \tilde{f}=0 \\
& \widetilde{\sigma}_{23}^{e}\left(d_{-}\right)-\widetilde{\sigma}_{23}^{e}\left(d_{+}\right)-\left\{i k_{x} \widetilde{T}_{x}+i k_{y} \widetilde{N}_{y}-\omega^{2} \rho^{h} h^{h} \widetilde{v}^{e}(d)\right\} * \tilde{f}=0 \\
& \widetilde{\sigma}_{33}^{e}\left(d_{-}\right)-\widetilde{\sigma}_{33}^{e}\left(d_{+}\right)-\left\{k_{x}^{2} \widetilde{M}_{x}-k_{y}^{2} \widetilde{M}_{y}+2 k_{x} k_{y} \widetilde{U}_{y}-\rho^{h} \omega^{2}\left[h^{h}+I\left(k_{x}^{2}+k_{y}^{2}\right)\right] \widetilde{w}^{e}(d)\right\} * \tilde{f}=0 .
\end{aligned}
$$

Où $\mathrm{d}_{\text {- et }} \mathrm{d}_{+}$correspondent aux faces inférieure et supérieure respectivement de la plaque mince et I est le moment d'inertie d'un hydrophone. La fonction $\tilde{\mathrm{f}}$ est représentative de la position des hydrophones dans 
l'élastomère et les fonctions $\vec{N}\left(N_{x}, N_{y}\right), \vec{T}\left(T_{x}, T_{y}\right), \vec{U}\left(U_{x}, U_{y}\right)$ et $\vec{M}\left(M_{x}, M_{y}\right)$ correspondent à l'effort normal, à l'effort tranchant longitudinal, au moment de torsion et au moment de flexion.

Les contraintes dans la partie supérieure de l'élastomère (au-dessus du plan contenant les hydrophones) se déduisent des conditions aux limites d'une part, entre l'eau et l'élastomère (continuité de la contrainte et đu déplacement normaux et annulation des contraintes tangentielles dans l'élastomère), et d'autre part entre l'élastomère et la plaque mince contenant les hydrophones (continuité du déplacement). De même, les contraintes dans la partie inférieure de l'élastomère (au-dessous du plan contenant les hydrophones) se déduisent des conditions aux limites, d'une part, entre l'élastomère et la plaque mince contenant les hydrophones (continuité du déplacement), et d'autre part, entre la coque et l'élastomère (continuité de la contrainte et du déplacement normaux et annulation des contraintes tangentielles dans l'élastomère).

En développant les produits de convolution des transformées de Fourier des efforts internes avec la fonction $\tilde{\mathrm{f}}$, et en introduisant les expressions des contraintes externes en fonction du déplacement dans les équations (1) à (3), le système à résoudre se met alors sous la forme :

$$
\begin{aligned}
& {\left[\mathbf{G}_{4 \rightarrow 6^{-1}}^{-1} \mathbf{H}_{4 \rightarrow 6}^{-1}\right] \stackrel{\widetilde{\mathrm{u}}}{\mathrm{e}}\left(\mathrm{k}_{\mathrm{x}}, \mathrm{k}_{\mathrm{y}}, \mathrm{d}\right)+\frac{i}{\omega^{2}} \frac{1^{2}}{\mathrm{D}^{2}} \mathbf{D}_{-\mathrm{d}}^{\mathrm{e}}\left[\mathbf{M}_{\text {contraintes }}^{\mathrm{e}}\right]^{-1} \sum_{\mathrm{n}=-\infty}^{+\infty} \sum_{\mathrm{m}=-\infty}^{+\infty}\left(\frac{\sin (\pi \mathrm{n} \mathrm{l} / \mathrm{D})}{\pi \mathrm{n} \mathrm{l} / \mathrm{D}} \frac{\sin (\pi \mathrm{ml} / \mathrm{D})}{\pi \mathrm{ml} / \mathrm{D}}\right.} \\
& \left.\mathrm{e}^{-2 i \pi n \mathrm{n}_{\mathrm{h}} / \mathrm{D}} \mathrm{e}^{-2 i \pi m \mathrm{~h}_{\mathrm{h}} / \mathrm{D}} \mathbf{M}^{\mathrm{h}}\left(\mathrm{k}_{\mathrm{x}}, \mathrm{k}_{\mathrm{y}} ; \mathrm{k}_{\mathrm{x}^{-}} \frac{2 \pi \mathrm{n}}{\mathrm{D}}, \mathrm{k}_{\mathrm{y}^{-}} \frac{2 \pi \mathrm{m}}{\mathrm{D}}\right) \widetilde{\mathrm{u}}\left(\mathrm{k}_{\mathrm{x}^{-}} \frac{2 \pi \mathrm{n}}{\mathrm{D}}, \mathrm{k}_{\mathrm{y}^{-}} \frac{2 \pi \mathrm{m}}{\mathrm{D}}, \mathrm{d}\right)\right)=\mathbf{G}_{1 \rightarrow 3}^{-1}\left(\begin{array}{c}
0 \\
0 \\
0 \\
\widetilde{w}^{\mathrm{c}}(0)
\end{array}\right) \text {. }
\end{aligned}
$$

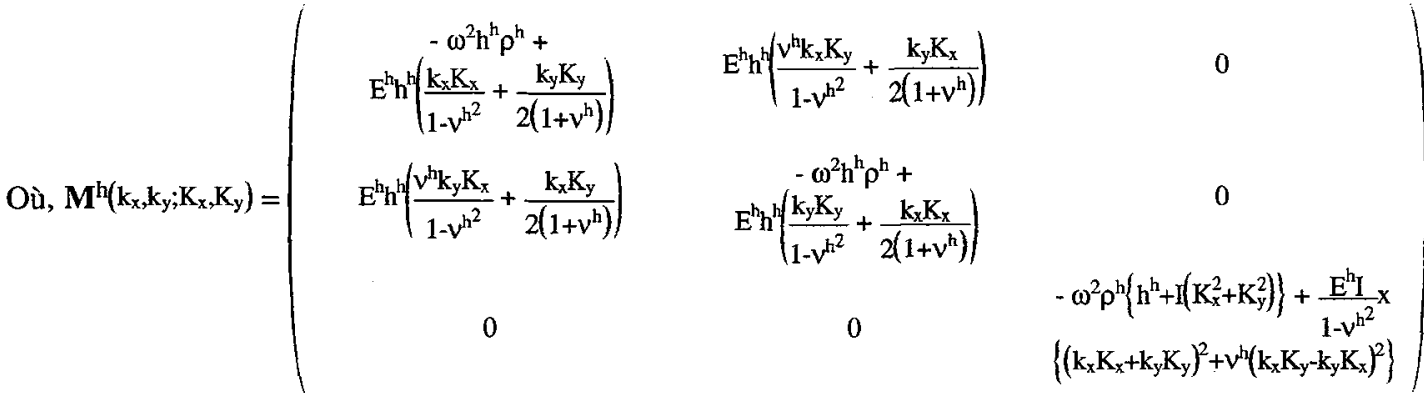

$\mathbf{G}_{1 \rightarrow 3}^{-1}, \mathbf{G}_{4 \rightarrow 6}^{-1}$ sont les matrices constituées des trois premières et trois dernières colonnes respectivement de la matrice inverse de $\mathbf{G}$ et $\mathbf{H}_{4 \rightarrow 6}^{-1}$ des trois dernières colonnes de l'inverse de la matrice de $\mathbf{H}$ :

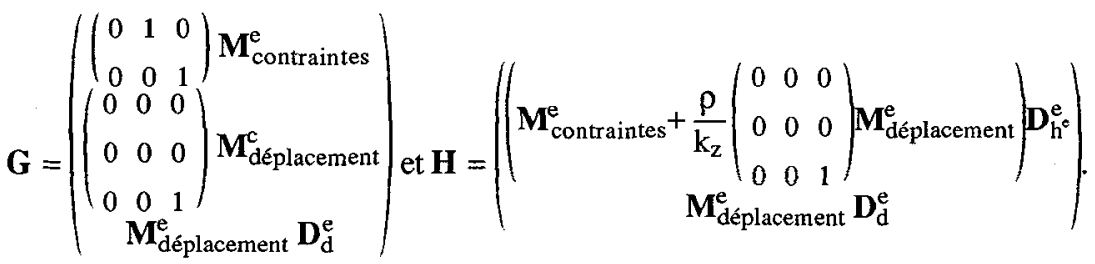

Et $\mathbf{D}_{\mathrm{h}^{c}}^{\mathrm{e}}, \mathbf{D}_{\mathrm{h}^{\mathrm{c}}}^{\mathrm{c}}$, sont des matrices diagonales de dimension 6 contenant les différences de marche des différentes ondes dans l'élastomère et la coque respectivement et $\mathbf{M}_{\text {déplacement }}^{\mathrm{c}}, \mathbf{M}_{\text {déplacement }}^{\mathrm{e}}, \mathbf{M}_{\text {contraintes }}^{\mathrm{e}}$ sont des matrices rectangles de dimensions $6 \times 3$ représentatives des caractéristiques mécaniques des deux matériaux.

La connaissance de la composante du champ électrique perpendiculaire au plan d'un hydrophone nécessite celle du tenseur des déformations par ses composantes $S_{1}, S_{2}$ et $S_{3}$. Ces dernières peuvent être déduites du champ de déplacement et de la contrainte normale dans la plaque mince correspondant à la surface moyenne contenant les hydrophones, calculés dans la phase précédente. 


\section{APPLICATION}

Les figures 2.a et 2.b présentent les amplitudes de deux des composantes du déplacement dans l'élastomère dans le plan des hydrophones. L'aspect en mosaïque est caractéristique de la présence des hydrophones. Cet effet est plus accentué sur les composantes tangentielles car les hydrophones résistent davantage à la traction qu'à la flexion.

Figure 2. Composantes du déplacement $u^{\mathrm{e}}(\mathrm{a})$ et $\mathrm{w}^{\mathrm{e}}(\mathrm{b})$ dans le plan des hydrophones.

a)

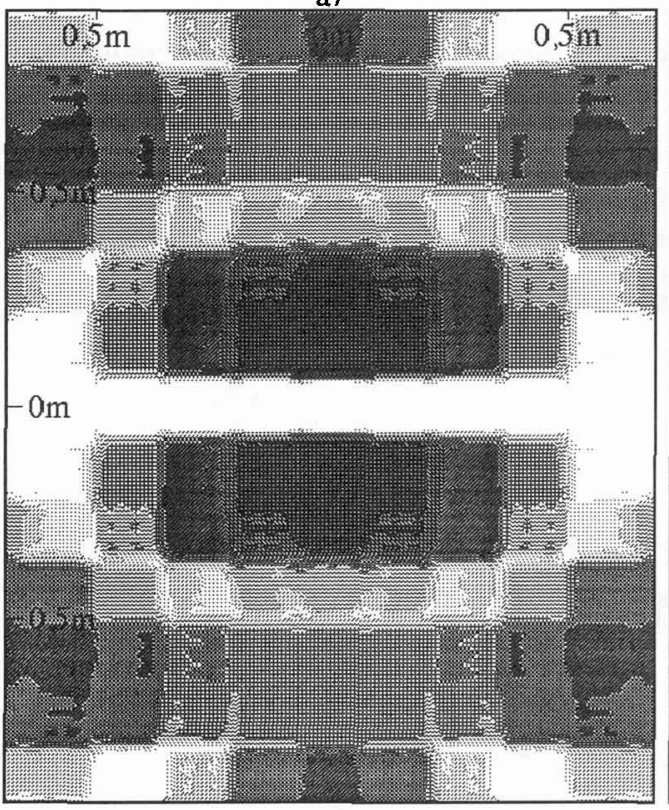

b)

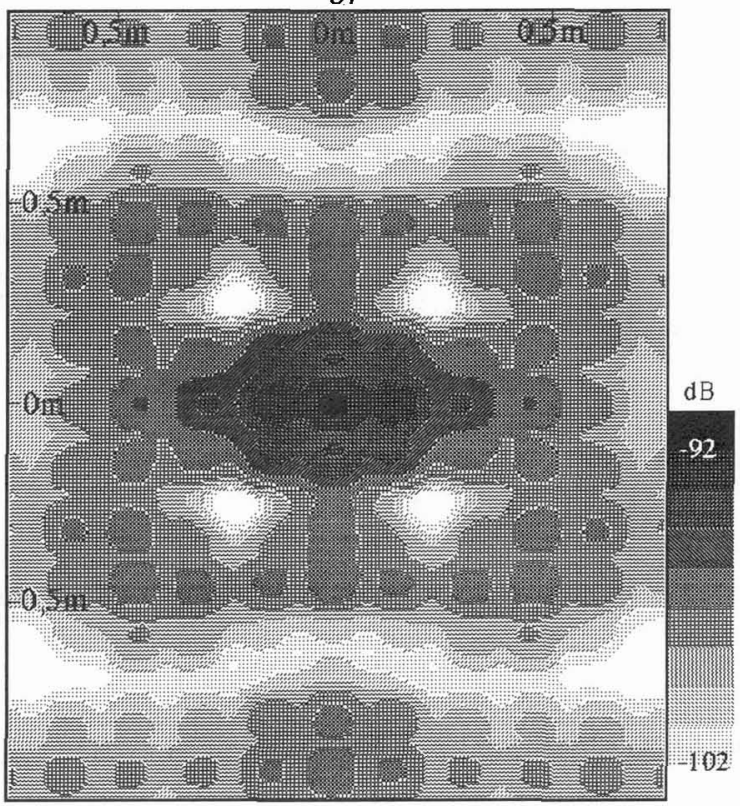

Si l'on souhaite optimiser le système global en réduisant au maximum l'intensité des potentiels engendrés aux bornes des hydrophones, nous pouvons alors faire les remarques suivantes :

- D'une façon générale, l'amplitude du potentiel associable à la déformation suivant la direction normale aux hydrophones est largement supérieure à celles des potentiels dus aux déformations tangentielles.

- Les résultats les plus performants sont observés pour un élastomère dissipatif présentant des caractéristiques proches de celles d'un fluide, celle de l'eau.

- L'augmentation de l'épaisseur des armatures externes des hydrophones rend l'antenne plus sensibles aux vibrations de la coque.

- Pour un type d'élastomère donné, il est possible d'optimiser la position des hydrophones, une position proche de la coque permet de minimiser l'amplitude du potentiel dû à la déformation normale.

\section{Remerciements}

Ce travail a été réalisé avec le support du CERDSM de la Direction des Constructions Navales de Toulon.

\section{Références}

[1] Burroughs C. B., Acoustic radiation from fluid-loaded infinite circular cylinders with doubly periodic ring supports, J. Acoust. Soc. Am., vol 75, N³, pp 715-722, mars 1984.

[2] Matteï P. O., Rayonnement acoustique des structures : méthodes d'équations intégrales de frontières, Thèse Univ. Marseille II, Inst. Méc. Marseille, juin 1991.

[3] Laroze S. \& Barrau J.-J., Mécanique des structures, Tomes 1 : Solides élastiques, plaques et coques, Ed. MASSON, 2 ème éd., 1991. 